\title{
Ezetimibe prevents the development of non-alcoholic fatty liver disease induced by high-fat diet in C57BL/6J mice
}

\author{
XIANG WANG $^{1}$, QIAOHUA REN ${ }^{1}$, TAO WU ${ }^{1}$, YONG GUO $^{2}$, YONG LIANG $^{3}$ and SUBO LIU $^{1}$ \\ Departments of ${ }^{1}$ Endocrinology and Metabolism, ${ }^{2}$ Urology and ${ }^{3}$ Preventive Medicine, \\ The First Hospital of Shijiazhuang City, Shijiazhuang, Hebei 050011, P.R. China
}

Received November 11, 2013; Accepted July 29, 2014

DOI: $10.3892 / \mathrm{mmr} .2014 .2623$

\begin{abstract}
There is currently no established treatment for non-alcoholic fatty liver disease (NAFLD), including its most extreme form, non-alcoholic steatohepatitis (NASH). Ezetimibe, an inhibitor of Niemann-Pick C1 Like 1-dependent cholesterol absorption, improves diet-induced hyperlipidemia and attenuates liver steatosis and insulin resistance. The aim of the present study was to determine whether ezetimibe treatment is able to inhibit the development of NAFLD, and to elucidate the underlying mechanism, using C57BL/6J (B6) mice maintained on a high-fat diet. Male B6 mice (20 weeks of age) were divided into the following two groups $(\mathrm{n}=7$ in each group): Mice fed a high-fat diet for four weeks and mice fed a high-fat diet with $0.0064 \%$ (wt/wt) ezetimibe $(5 \mathrm{mg} / \mathrm{kg} / \mathrm{day})$ for four weeks. Administration of ezetimibe significantly reduced liver steatosis and fibrosis. Ezetimibe reduced serum cholesterol, hepatic fat accumulation and insulin resistance in the liver of mice fed the high-fat diet. Furthermore, ezetimibe significantly reduced hepatic mRNA expression of Accl and $S c d l$, which are involved in hepatic fatty acid synthesis. Ezetimibe significantly reduced hepatic $C d 36$ gene expression, upregulation of which is significantly associated with insulin resistance, hyperinsulinemia and increased steatosis. The protein expression of SKP2, a viable therapeutic target in human cancer, was also reduced by ezetimibe. These findings suggest that ezetimibe may be an effective therapy for high fat-induced NAFLD, including NASH.
\end{abstract}

Correspondence to: Dr Xiang Wang, Department of Endocrinology and Metabolism, The First Hospital of Shijiazhuang City, 36 Fan-xi Road, Shijiazhuang, Hebei 050011, P.R. China

E-mail: wangxiang801028@163.com

Abbreviations: MTP, microsomal triglyceride transfer protein; NASH, non-alcoholic steatohepatitis; NAFLD, non-alcoholic fatty liver disease; HCC, hepatocellular carcinoma; EZ, ezetimibe; HF, high fat diet; TG, triglyceride; Chol, cholesterol; B6 mouse, C57BL/6J mouse; ALT, alanine aminotransferase

Key words: non-alcoholic fatty liver disease, hepatocellular carcinoma, liver steatosis, insulin resistance, ezetimibe

\section{Introduction}

Non-alcoholic fatty liver disease (NAFLD) is a clinicopathological syndrome in which the severity can range from simple fatty liver to non-alcoholic steatohepatitis (NASH), cirrhosis and hepatocellular carcinoma, and is observed in patients with no history of excessive alcohol consumption (1). A previous study has indicated that NAFLD can coexist with type 2 diabetes mellitus, obesity and dyslipidemia. In particular, hyperlipidemia and insulin resistance were implicated in the initiation and progression of NAFLD (2). Currently, an increase in energy consumption by exercise and/or a reduction in energy intake are accepted methods for the prevention of NAFLD. No standard treatments are currently used to reverse NAFLD, and effective medical interventions have focused on diet control and exercise. There remains a requirement for the development of effective pharmacological agents, due to the increasing prevalence of NAFLD.

Ezetimibe remains the most widely used first-line drug for the treatment of hypercholesteremia. Ezetimibe exerts its effect predominantly by inhibiting cholesterol absorption and it was demonstrated to block Niemann-Pick C1 Like 1-mediated cholesterol absorption at the brush border of the intestine and the liver (3). In addition to improving hypercholesterolemia in patients with dyslipidemia, attention has recently been drawn to its potential attenuation of liver steatosis $(4,5)$. It has been shown that Ezetimibe may exert these effects by reducing Srebp-1c expression in mice fed a high-fat diet (6). Another study demonstrated that ezetimibe was effective for reducing serum low-density lipoprotein cholesterol levels resistant to lifestyle intervention in patients with non-alcoholic fatty liver disease (7). Hepatic steatosis, induced by a high-fat diet, but not a high-fructose diet, was inhibited by ezetimibe administration (8). It has also been reported that hepatic iron levels in mice fed a high-fat diet are increased following treatment with ezetimibe (9). Despite these notable findings, mechanisms by which ezetimibe administration ameliorates hepatic steatosis, insulin resistance and obesity remain largely unexplored.

NAFLD is considered as the hepatic manifestation of the metabolic syndrome that is closely associated with obesity, hyperlipidemia, type 2 diabetes mellitus and insulin resistance. The initiation and progression of metabolic syndrome (MS) is mainly associated with the consumption of high-fat diets and/or high-carbohydrate diets. Epidemiological studies 
suggest that consumption of high-fat diets ( $\geq 30 \%$ of energy from fat) is associated with a high prevalence of being overweight, central obesity and MS $(10,11)$. Rodents fed a high-fat diet closely mimic a number of the features observed in humans with NAFLD, and present with obesity, impaired glucose tolerance, dyslipidemia and fat accumulation in the liver $(12,13)$. In the current study, the effects of ezetimibe on an HF-induced mouse model C57BL/6J (B6) for NAFLD were investigated.

\section{Materials and methods}

Animals. Male B6 mice (14 weeks old) were purchased from the Hebei Medical University, Center for Animal Experimentation (Shijiazhuang, China) and were maintained in the animal facilities of Hebei Medical University with standard animal care procedures based on the institutional guidelines. The mice were fed a normal laboratory diet $(22.3 \%$ protein, $6.2 \%$ fat, $3.0 \%$ fiber, $6.5 \%$ ash and $47.8 \%$ complex carbohydrate) with free access to water, and were housed with a regular 12-h light/dark cycle according to the Hebei Medical University Guidelines for the Care and Use of Laboratory Animals. Following acclimatization for two weeks, B6 mice were fed a high-fat chow (High-Fat Diet 32; CLEA Japan, Inc., Tokyo, Japan) for four weeks, then the mice were divided into two groups ( $n=7 /$ group); those fed the high-fat chow for four weeks (HF group), and those fed the high-fat chow with $0.0064 \% \mathrm{wt} / \mathrm{wt}$ ezetimibe $(5 \mathrm{mg} / \mathrm{kg} /$ day) for four weeks (HF+EZ group). After 16-h fasting, all mice were sacrificed under anaesthesia by intraperitoneal administration of pentobarbital (60 mg/kg body weight; Nembutal; Dainippon Sumimoto Pharma Co., Ltd., Osaka Japan) and medetomidine (0.3 mg/kg body weight; Domitor; Meiji Seika Kaisha, Ltd., Tokyo, Japan). The ezetimibe was provided by Merck Sharp \& Dohme (Whitehouse Station, NJ, USA).

Phenotype determination. Alanine aminotransferase (ALT), total serum cholesterol and triglyceride (TG) were measured as described previously (14).

Measurement of liver triglyceride. Liver triglyceride content was analyzed using a Triglyceride Quantification Kit (\#ab65336; Abcam, Cambridge, MA, USA) as previously described (14).

Intraperitoneal glucose tolerance test (ipGTT). The mice were given an ipGTT ( $2 \mathrm{~g}$ glucose/kg body weight) subsequent to overnight fasting. The glucose levels were measured after fasting prior to glucose administration ( $0 \mathrm{~min}$ ), and at 30,60, 90 and 120 min post-glucose load.

Histological examination of liver. To study histological changes, liver tissue samples were formalin-fixed and paraffin-embedded (Santa Cruz Biotechnology, Inc., Dallas, TX, USA) and subjected to hematoxylin-eosin (H\&E; Biocare Medical, LLC., Concord, CA, USA) and Sirius red (Direct Red 80; Sigma-Aldrich, St. Louis, MO, USA) staining. All the images were acquired and analyzed using a BZ-8000 Fluorescence Microscope (Keyence Corporation, Osaka, Japan). H\&E-stained sections and Sirius red-stained sections were graded according to the NASH activity score and the
Table I. Characteristics of C57BL/6J mice fed a high-fat diet with or without ezetimibe.

\begin{tabular}{lcc}
\hline & \multicolumn{2}{c}{ C57BL/6J } \\
\cline { 2 - 3 } Variable & HF & HF+EZ \\
\hline Starting body weight (g) & $30.0 \pm 1.6$ & $30.0 \pm 1.8$ \\
Final body weight (g) & $35.4 \pm 2.0$ & $28.8 \pm 2.1^{\mathrm{b}}$ \\
Liver weight (mg) & $1026 \pm 63$ & $858 \pm 47^{\mathrm{b}}$ \\
Liver weight/body weight & $0.030 \pm 0.001$ & $0.032 \pm 0.003$ \\
NASH activity score & $2.3 \pm 0.7$ & $1.0 \pm 0.5^{\mathrm{b}}$ \\
Fibrosis score & $1.0 \pm 0.2$ & $0.6 \pm 0.1^{\mathrm{b}}$ \\
Serum ALT (IU/L) & $35.7 \pm 8.5$ & $30.9 \pm 7.2$ \\
Serum total cholesterol (mg/dl) & $119.6 \pm 11.3$ & $90.0 \pm 28.5^{\mathrm{a}}$ \\
Serum triglyceride (mg/dl) & $14.7 \pm 4.6$ & $12.0 \pm 3.7$ \\
Liver TG content (mg/g liver) & $16.2 \pm 1.4$ & $13.5 \pm 1.5^{\mathrm{a}}$ \\
\hline
\end{tabular}

The data are expressed as the means \pm standard deviation; $n=7$. ${ }^{\mathrm{a}} \mathrm{P}<0.05$ and ${ }^{\mathrm{b}} \mathrm{P}<0.01, \mathrm{HF}$ vs. HF+EZ. HF, high-fat chow; EZ, ezetimibe; NASH, non-alcoholic steatohepatitis; ALT, alanine aminotransferase; TG, triglyceride.

fibrosis score as previously described $(15,16)$. The evaluation was performed by two experienced pathologists who were blinded to the treatments that the mice had received, according to methods previously described (16).

Hepatic gene expression analysis. Total RNA was extracted from frozen liver samples using Isogen (Nippon Gene Co., Ltd., Tokyo, Japan), and cDNA was synthesized using a High Capacity cDNA Reverse Transcription kit (Applied Biosystems Life Technologies, Carlsbad, CA, USA). Quantitative polymerase chain reaction (qPCR) was performed using TaqMan ${ }^{\circledR}$ Gene Expression Master Mix (Applied Biosystems Life Technologies) with a previously described method (17). The expression of the following genes was evaluated with probes from Applied Biosystems Life Technologies as follows: Accl (Mm01304282_m1) for acetyl-coenzyme A (CoA) carboxylase 1; Srebfl (Mm00550338_m1) for sterol regulatory element binding protein (SREBP)-1c; Scdl (Mm00772290) for stearoyl-CoA desaturase 1; Fasn (Mm00662319_m1) for fatty acid synthase; Srebf2 (Mm01306289_m1) for SREBP-2; Cd36 (Mm00432403) for the fatty acid translocase (FAT); Dgat2 (Mm00499530) for diacylglycerol O-acetyltransferase 2; Bax (Mm00432448_m1) for Bcl-2 associated X protein; Bcl-2 (Mm00477631_m1) for B cell lymphoma-2; Cptla (Mm00550438_m1) for carnitine palmitoyltransferase 1A; Ppara (Mm00440939_m1) for peroxisome proliferator-activated receptor $\alpha$; $A p o B$ (Mm01545164_m1) for apolipoprotein B; Mttp (Mm00435015_m1) for microsomal triglyceride transfer protein (MTP); Ccl2 (Mm00441242_m1) for chemokine (C-C motif) ligand 2; Emr1 (Mm00802530_m1) for cell surface glycoprotein F4/80; Tnf (Mm00443258_m1) for tumor necrosis factor- $\alpha$; and Tgfbl (Mm00441724_m1) for transforming growth factor $\beta-1$. All experiments were performed in duplicate and 

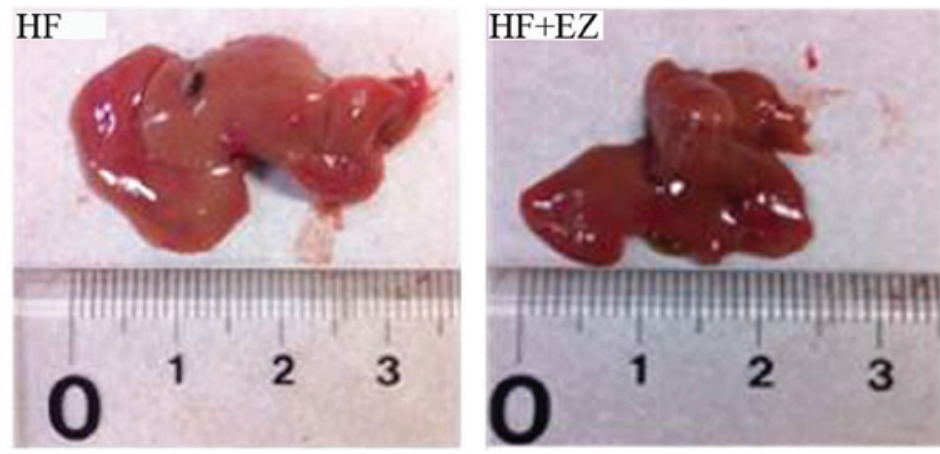

Figure 1. Macroscopic findings observed following short-term treatment with ezetimibe (EZ) in C57BL/6J mice fed a high-fat chow (HF).
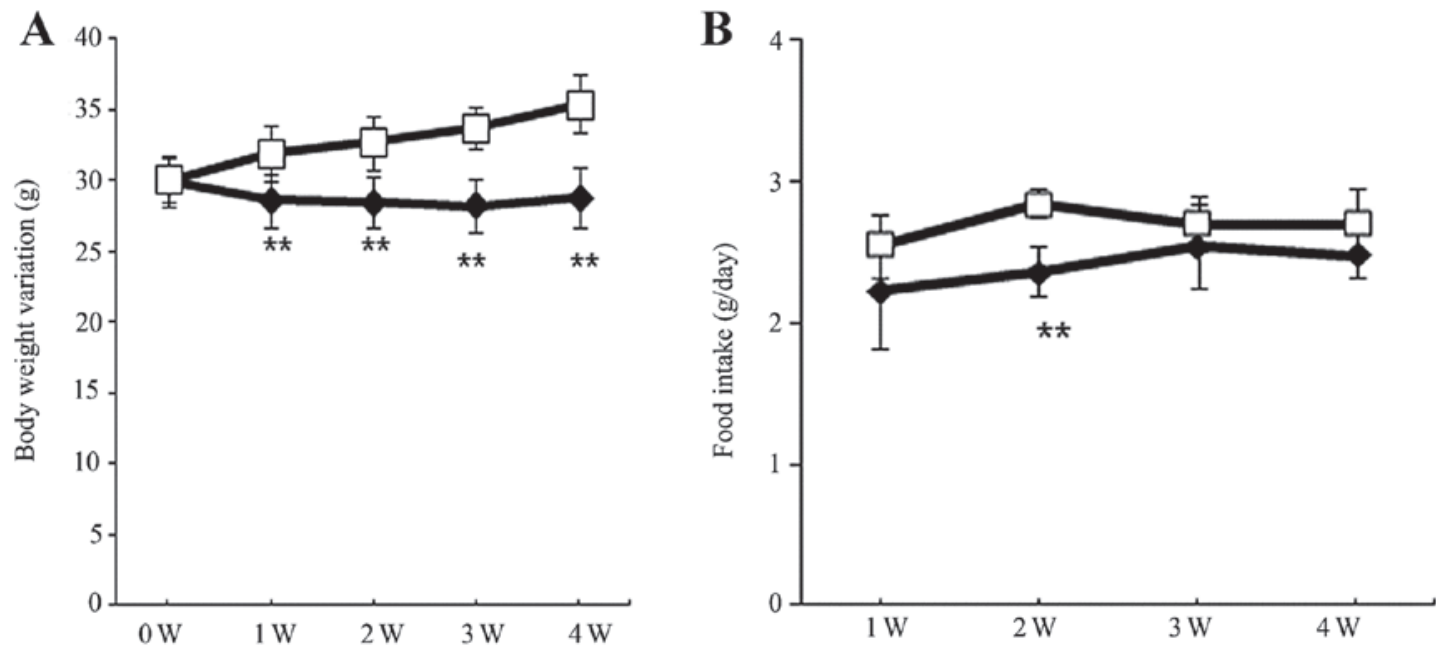

Figure 2. Food consumption and body weight variation of C57BL/6J mice fed high-fat chow. Variations in (A) body weight and (B) food consumption. White squares, mice fed high-fat chow; black circles, mice fed high-fat chow plus ezetimibe. The data are expressed as the means \pm standard deviation $(\mathrm{n}=7)$. ${ }^{* *} \mathrm{P}<0.01$, vs. HF group. W, week.

all gene expression levels were normalized to Hprtl expression (Mm00446968_m1).

Western blot analysis. Liver samples were collected and proteins were separated by SDS-PAGE (10\% MiniProtean ${ }^{\circledR}$ TGX $^{\mathrm{TM}}$ gel and Mini-Protean ${ }^{\circledR}$ Tetra Cell Mini Trans-Blot module; Bio-Rad Laboratories, Hercules, CA, USA), and blotted onto polyvinylidine fluoride membranes (Bio-Rad Laboratories). The blots were incubated with polyclonal anti-rabbit SKP2 (L70; \#4313; 1:1,000) or $\beta$-actin primary antibodies (\#4967, 1:1,000; both from Cell Signaling Technology, Inc., Danvers, MA, USA) overnight at $4^{\circ} \mathrm{C}$ using slow rocking and the horseradish peroxidase (HRP)-conjugated secondary antibody, anti-rabbit IgG-HRP (1:1,500; Cell Signaling Technology, Inc.). Thereafter, the membranes were visualized by enhanced chemiluminescence, and the signals were quantified as previously described (16).

Statistical analysis. All data are expressed as the means \pm standard deviation. Statistical comparisons were made using the two independent-samples t-test and Mann-Whitney $\mathrm{U}$ test. $\mathrm{P}<0.05$ was considered to indicate a statistically significant difference. All statistical analyses were performed using SPSS for Windows, version 18 (SPSS, Inc., Chicago, IL, USA).

\section{Results}

Physiological characteristics. The livers of HF mice were markedly enlarged and exhibited a paler color as compared with the livers of the HF+EZ group (Fig. 1, Table I) The food consumption and body weight of the two groups were monitored throughout the observation period. Although the baseline body weight was similar between the two groups (Table I, Fig 2A), the body weight was significantly lower in the HF+EZ group as compared with the HF group during weeks 1-4 following ezetimibe treatment $(\mathrm{P}<0.01$; Fig. $2 \mathrm{~A})$. Food consumption was similar between $\mathrm{HF}$ and HF+EZ groups (Fig. 2B). The final body and liver weights were significantly lower in the HF+EZ group than in the HF group $(\mathrm{P}<0.01$; Table I). Liver TG content was significantly lower in the HF+EZ group, as compared with that of the HF group $(\mathrm{P}<0.05$; Table I).

Histological changes in the liver. Lipid deposits in the liver were smaller in the HF+EZ group as compared with those in the HF group (Fig. 3A). Regarding the NASH activity score, 

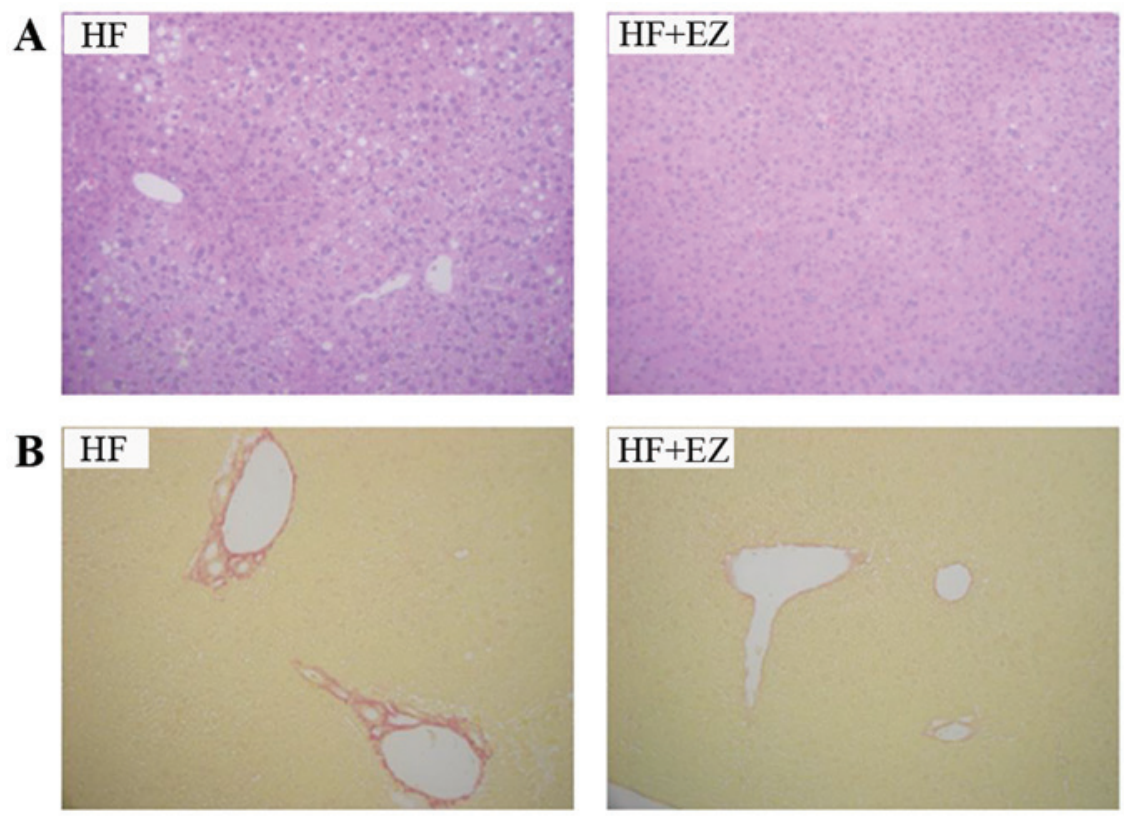

Figure 3. Histological appearance of the liver from C57BL/6J mice fed high-fat chow (HF). Liver sections from mice fed HF and HF plus ezetimibe (EZ). (A) Hematoxylin and eosin staining indicating reduced lipid deposits and (B) Sirius red staining indicating reduced fibrosis in the HF+EZ group, as compared with the HF group. Magnification, x200.

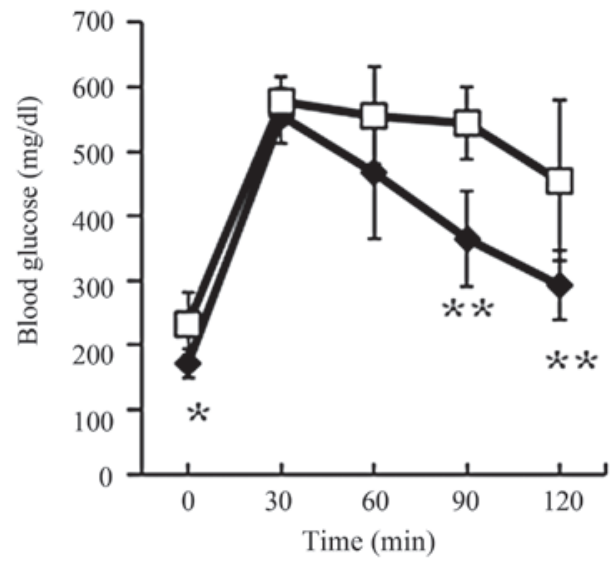

Figure 4. Effect of ezetimibe (EZ) on glucose metabolism. Glucose levels during an intraperitoneal glucose tolerance test were analyzed in C57BL/6J mice fed high-fat chow. White squares, mice fed HF; black rhombuses, mice fed HF plus ezetimibe. The data are expressed as the mean \pm standard deviation $(\mathrm{n}=7) .{ }^{*} \mathrm{P}<0.05$ and ${ }^{* *} \mathrm{P}<0.01$, vs. HF group.

the HF group had a total score of $2.3 \pm 0.7$, while the HF+EZ group had a total score of $1.0 \pm 0.5$, indicating a significant difference between the two groups $(\mathrm{P}<0.01$; Table I). Sirius red staining of the liver revealed that the HF+EZ group exhibited a lower degree of liver fibrosis as compared with the HF group (Fig. 3B). The fibrosis score was significantly different between the two groups $(1.0 \pm 0.2$ in the HF group vs. $0.6 \pm 0.1$ in the $\mathrm{HF}+\mathrm{EZ}$ group; $\mathrm{P}<0.01$; Table I).

Glucose tolerance. The fasting glucose level in the $\mathrm{HF}+\mathrm{EZ}$ group was significantly lower than that of the HF group ( $\mathrm{P}<0.05$; Fig. 4). In addition, the blood glucose level at 90 and 120 min during the ipGTT were significantly lower in the HF+EZ group as compared with the HF group $(\mathrm{P}<0.01$; Fig. 4).
Serum biochemical markers. Serum total cholesterol levels in the HF+EZ group were significantly lower than that of the HF group $(\mathrm{P}<0.05$; Table I). Serum ALT and TG levels in the HF+EZ group were not significantly lower than those in the HF group (Table I).

Hepatic gene expression for lipid metabolism, inflammation, fibrosis and apoptosis. The mRNA levels of specific hepatic lipogenesis-related genes were evaluated, including the following: Acc1, Srebf1, Srebf2, Scd1, Dagt2, Cd36 and Fasn. Hepatic expression levels of Accl, Scdl and Cd36 were significantly lower in the HF+EZ group, compared with the HF group ( $\mathrm{P}<0.05$; Fig. 5), while those of Srebf 2 were significantly higher $(\mathrm{P}<0.01)$. Notably, the hepatic expression level of Mttp was also significantly lower in the HF+EZ group, as compared with the HF group $(\mathrm{P}<0.05)$. Expression levels of genes associated with lipid catabolism, fibrosis and apoptosis were not significantly different between the two groups. Regarding the expression of genes involved in inflammation, Ccl2 and Tnf were slightly lower in the HF+EZ group than in the HF group, however this was not significant, and there was no difference in the expression of Emrl between the two groups (Fig. 5).

Western blot analysis of SKP2. The protein expression level of SKP2 in the HF+EZ group was significantly lower than that in the HF group ( $\mathrm{P}<0.01$; Fig. 6$)$.

\section{Discussion}

NAFLD is a growing public health concern in developed and developing countries due to the increasing prevalence of obesity, diabetes and the metabolic syndrome induced by the excessive consumption of high-fat and cholesterol-containing diets and the lack of exercise in the general population. 

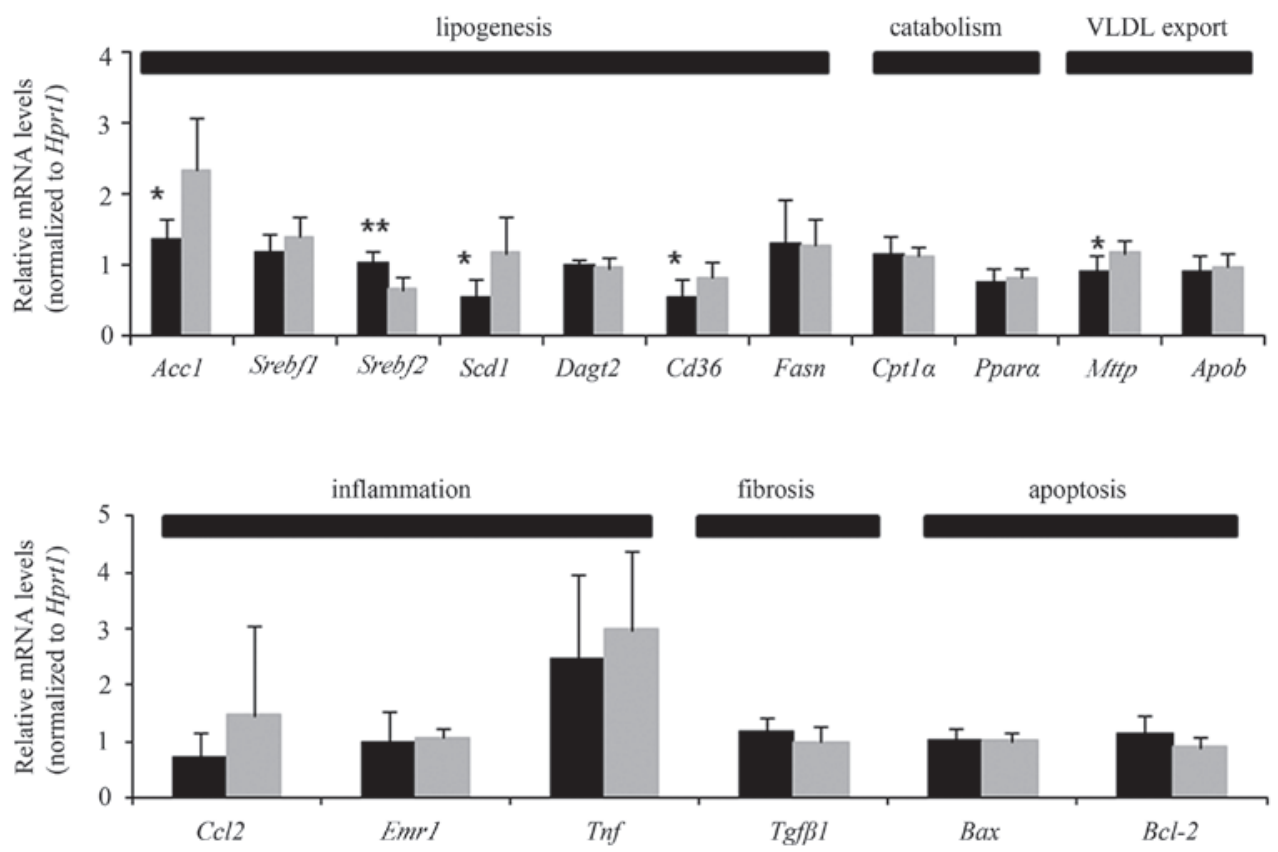

Figure 5. Effect of ezetimibe (EZ) treatment on hepatic gene expression. Gene expression analysis by quantitative polymerase chain reaction from the livers of mice fed high-fat chow (HF) plus EZ treatment (black bars) and HF group (gray bars). The mRNA levels are expressed relative to those in the HF group, as the mean \pm standard deviation, following normalization against the expression of $H p r t 1 \mathrm{mRNA}(\mathrm{n}=7)$. ${ }^{*} \mathrm{P}<0.05$ and ${ }^{* *} \mathrm{P}<0.01$, vs. HF group.

A

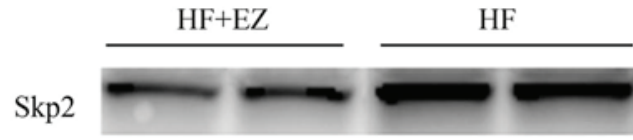

$\beta$-actin

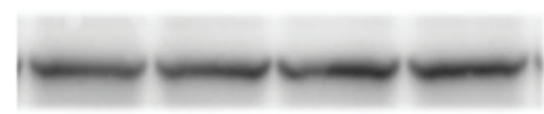

B

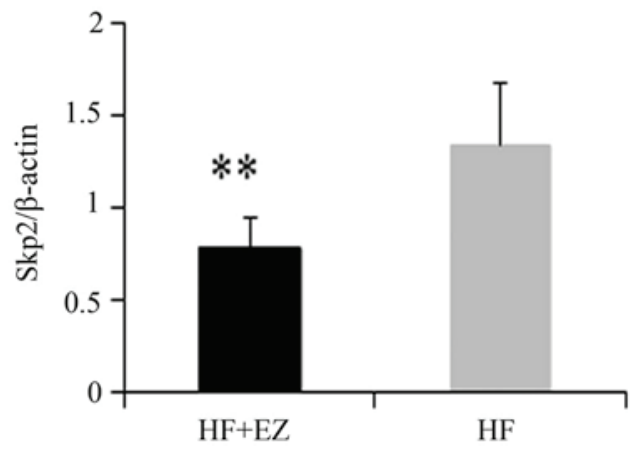

Figure 6. Effect of ezetimibe (EZ) treatment on hepatic protein expression. (A) Western blot analysis of SKP2 in livers from the high fat chow (HF) and $\mathrm{HF}+\mathrm{EZ}$ groups, and (B) densitometric quantification of SKP2/ $\beta$-actin expression in livers from the HF+EZ (black bars) and HF (gray bars) groups. The data represent the means \pm standard deviation $(n=7) .{ }^{* *} \mathrm{P}<0.01$, vs. HF group.

In the present study, the effects of ezetimibe on NAFLD were investigated using a high-fat induced mouse model. The results demonstrated that ezetimibe significantly reduced the NASH activity and fibrosis score in mice fed the high-fat diet compared with the untreated mice, indicating favorable effects of ezetimibe on liver steatosis and fibrosis. In addition, ezetimibe improved serum cholesterol, hepatic fat accumulation and insulin resistance in the livers of mice fed a high-fat diet. Furthermore, ezetimibe significantly reduced mRNA levels of $A c c l, S c d l$ and $C d 36$ in the liver, which are all associated with hepatic lipogenesis and free fatty acid transportation. The level of SKP2 protein upregulation, which induces uncontrolled cell proliferation and tumor progression, was also reduced by ezetimibe.

Ezetimibe administration reduced the body and liver weights. The current study considered the possibility that the effect of ezetimibe may be mediated by food intake, as reduced food intake would significantly affect body weight, and therefore influence hepatic steatosis. However, the reduced body and liver weights were not associated with reduced food consumption in the present study, and weight-specific food intake was indicated to be similar between the two groups. This suggests that ezetimibe directly protected against obesity and hepatic steatosis, independent of food intake (18).

A previous study in C57BL/6 mice demonstrated that fat overconsumption is key in the etiology of hepatic steatosis (19). Another study suggested that lipid accumulation in the liver of mice and rats can be induced by a high-fat diet (20). Long-term fat overconsumption may increase the risk of insulin resistance and obesity, which enhance susceptibility to NAFLD. In rodent models and humans, hepatic steatosis is consistently associated with the development of hepatic insulin resistance (2). In the current study, ezetimibe ameliorated insulin resistance in mice fed the high-fat diet, and significantly altered fasting glucose in addition to glucose levels at 90 and $120 \mathrm{~min}$ of the ipGTT, which strongly indicated that ezetimibe improved insulin sensitivity. Ezetimibe administration may inhibit high fat-induced insulin resistance by reducing intestinal fat absorption and weight gain, rather than via downregulation of Srebf 1 as suggested previously $(6,21)$. 
CD36 functions in the development of hepatic steatosis in rodents, and is the most well-characterized free fatty acid transporter. Thus far, the significance of CD36 in human liver diseases remains unclear. Hepatic expression of CD36 is abnormally increased in non-alcoholic fatty liver disease, and a previous study indicated that hepatic CD36 upregulation was significantly associated with insulin resistance, hyperinsulinemia and increased steatosis in patients with NAFLD (22). Hepatic CD36 expression is normally low, but its expression is increased in rodents with fatty liver (23). Additionally, another study demonstrated that CD36 mRNA levels increased concomitantly with hepatic TG content in a number of animal models of fatty liver $(24,25)$. Notably, experimental amelioration of steatosis in mice was accompanied by hepatic CD36 downregulation $(26,27)$. Modulation of CD36 expression in hepatocytes may prove useful for the prevention or treatment of liver fat accumulation in patients with NAFLD. In the present study, ezetimibe significantly reduced CD36 gene expression in the liver. Hence, ezetimibe may ameliorate hepatic insulin resistance in addition to dyslipidemia and hepatic steatosis, partly via a pathway involving CD36 in high-fat diet-induced B6 mouse models of NAFLD.

SREBP-1c is a key transcriptional activator of lipogenesis, and is responsible for regulating genes involved in lipogenesis, including Accl, Fas and Scdl (5). In the present study, ezetimibe treatment reduced the mRNA expression levels of Accl and $S c d l$, which were correlated with hepatic lipogenesis, and upregulated the gene expression of Srebf2, as previously reported (6). Inhibition of cholesterol absorption may occur by ezetimibe-activated hepatic expression of SREBP-2, which is established as a key regulator of cholesterol synthesis and uptake (28). The hepatic mRNA expression level of Mttp was increased in the HF group compared with the level in the $\mathrm{HF}+\mathrm{EZ}$ group in the present study, suggesting a compensatory mechanism to release excess lipid as VLDL, as previously suggested (29).

Overexpression of SKP2, a positive regulator of G1-to-S phase transition, has been observed in numerous cases of human cancer, including hepatocellular carcinoma (HCC) $(30,31)$. Previous reports have demonstrated in various types of cancer, that SKP2 mRNA or protein expression is increased compared with normal tissues (32-35). Uncontrolled SKP2 upregulation may favor cell transformation in vitro and tumor progression $(36,37)$. It has been reported that a mouse knockout for $S k p 2$ results in a reduction of cell proliferation and mouse body size (38). Another study indicated that silencing of $S k p 2$ by siRNA in HuH7 and HepG2 cells led to growth restraint, enhanced apoptosis, and a rise in protein levels of cell cycle inhibitors, with consequent reduction of their ubiquitination (30). Other studies have indicated that $S k p 2$ serves as an oncogene in HCC and thus is upregulated by increased transcriptional activity $(39,40)$. Therefore, the $S k p 2$ gene may be a therapeutic target for NAFLD-related HCC. In the present study, the protein expression of SKP2 was reduced by ezetimibe administration, providing a clue that ezetimibe may prevent the progression of NAFLD-related HCC through downregulation of SKP2 protein expression. However, further research is required to confirm this contention.

In conclusion, ezetimibe administration in an HF-induced model of NAFLD resulted in lower serum cholesterol levels, and amelioration of glucose tolerance, histological lesions and hepatic expression of lipogenesis-related genes. In addition, B6 mice that received ezetimibe treatment presented lower $C d 36$ gene expression in the liver, suggesting ezetimibe may ameliorate hepatic insulin resistance in addition to dyslipidemia and hepatic steatosis, in part via a pathway involving $C d 36$. Furthermore, the protein level of SKP2, a therapeutic target for NAFLD-related HCC, was reduced by ezetimibe administration. These data suggest that ezetimibe may have the potential to be used as an effective drug for NAFLD and NAFLD-related HCC.

\section{Acknowledgements}

The authors would like to thank Ms. Chunhuan Ma for her technical assistance.

\section{References}

1. Wieckowska A, McCullough AJ and Feldstein AE: Noninvasive diagnosis and monitoring of nonalcoholic steatohepatitis: present and future. Hepatology 46: 582-589, 2007.

2. Deushi M, Nomura M, Kawakami A, et al: Ezetimibe improves liver steatosis and insulin resistance in obese rat model of metabolic syndrome. FEBS Lett 581: 5664-5670, 2007.

3. Altmann SW, Davis HR Jr, Zhu LJ, et al: Niemann-Pick C1 Like 1 protein is critical for intestinal cholesterol absorption. Science 303: 1201-1204, 2004.

4. Zheng S, Hoos L, Cook J, et al: Ezetimibe improves high fat and cholesterol diet-induced non-alcoholic fatty liver disease in mice. Eur J Pharmacol 584: 118-124, 2008.

5. Matono T, Koda M, Tokunaga S, et al: Therapeutic effects of ezetimibe for non-alcoholic steatohepatitis in fatty liver shionogi-ob/ob mice. Hepatol Res 41: 1240-1248, 2011.

6. Muraoka T, Aoki K, Iwasaki T, et al: Ezetimibe decreases SREBP-1c expression in liver and reverses hepatic insulin resistance in mice fed a high-fat diet. Metabolism 60: 617-628, 2011.

7. Oza N, Takahashi H, Eguchi Y, et al: Efficacy of ezetimibe for reducing serum low-density lipoprotein cholesterol levels resistant to lifestyle intervention in patients with non-alcoholic fatty liver disease. Hepatol Res 44: 812-817, 2014.

8. Ushio M, Nishio Y, Sekine O, et al: Ezetimibe prevents hepatic steatosis induced by a high-fat but not a high-fructose diet. Am J Physiol Endocrinol Metab 305: E293-E304, 2013.

9. Kishino Y, Tanaka Y, Ikeda T, et al: Ezetimibe increases hepatic iron levels in mice fed a high-fat diet. J Pharmacol Exp Ther 345: 483-491, 2013.

10. George V, Tremblay A, Després JP, Leblanc C and Bouchard C: Effect of dietary fat content on total and regional adiposity in men and women. Int J Obes 14: 1085-1094, 1990.

11. Tucker LA and Kano MJ: Dietary fat and body fat: a multivariate study of 205 adult females. Am J Clin Nutr 56: 616-622, 1992.

12. Takahashi Y, Soejima Y and Fukusato T: Animal models of nonalcoholic fatty liver disease/nonalcoholic steatohepatitis. World J Gastroenterol 18: 2300-2308, 2012.

13. Buettner R, Schölmerich J and Bollheimer LC: High-fat diets: modeling the metabolic disorders of human obesity in rodents. Obesity (Silver Spring) 15: 798-808, 2007.

14. Wang X, Sugimoto K, Fujisawa T, et al: Novel effect of ezetimibe to inhibit the development of non-alcoholic fatty liver disease in Fatty Liver Shionogi mouse. Hepatol Res 44: 102-113, 2014.

15. Kleiner DE, Brunt EM, Van Natta M, et al; Nonalcoholic Steatohepatitis Clinical Research Network: Design and validation of a histological scoring system for nonalcoholic fatty liver disease. Hepatology 41: 1313-1321, 2005.

16. Shindo N, Fujisawa T, Sugimoto K, et al: Involvement of microsomal triglyceride transfer protein in nonalcoholic steatohepatitis in novel spontaneous mouse model. J Hepatol 52: 903-912, 2010.

17. Oze-Fukai A, Fujisawa T, Sugimoto K, et al: A novel mouse model for type 2 diabetes and non-alcoholic fatty liver disease: spontaneous amelioration of diabetes by augmented beta cell mass. Endocr J 56: 227-234, 2009. 
18. Jung HY, Kim YH, Kim IB, et al: The Korean mistletoe (Viscum album coloratum) extract has an antiobesity effect and protects against hepatic steatosis in mice with high-fat diet-induced obesity. Evid Based Complement Alternat Med 2013: 168207, 2013.

19. Jia L, Betters JL and Yu L: Niemann-pick C1-like 1 (NPC1L1) protein in intestinal and hepatic cholesterol transport. Annu Rev Physiol 73: 239-259, 2011.

20. Oosterveer MH, van Dijk TH, Tietge UJ, et al: High fat feeding induces hepatic fatty acid elongation in mice. PLoS One 4 e6066, 2009

21. Jia L, Ma Y, Rong S, et al: Niemann-Pick C1-Like 1 deletion in mice prevents high-fat diet-induced fatty liver by reducing lipogenesis. J Lipid Res 51: 3135-3144, 2010.

22. Miquilena-Colina ME,Lima-CabelloE, Sánchez-Campos S, et al: Hepatic fatty acid translocase CD36 upregulation is associated with insulin resistance, hyperinsulinaemia and increased steatosis in non-alcoholic steatohepatitis and chronic hepatitis $\mathrm{C}$. Gut 60: 1394-1402, 2011.

23. Inoue M, Ohtake T, Motomura W, et al: Increased expression of PPARgamma in high fat diet-induced liver steatosis in mice. Biochem Biophys Res Commun 336: 215-222, 2005.

24. Buqué X, Martínez MJ, Cano A, et al: A subset of dysregulated metabolic and survival genes is associated with severity of hepatic steatosis in obese Zucker rats. J Lipid Res 51: 500-513, 2010.

25. Degrace P, Moindrot B, Mohamed I, et al: Upregulation of liver VLDL receptor and FAT/CD36 expression in LDLR-/apoB100/100 mice fed trans-10,cis-12 conjugated linoleic acid J Lipid Res 47: 2647-2655, 2006.

26. Liu LF, Purushotham A, Wendel AA and Belury MA: Combined effects of rosiglitazone and conjugated linoleic acid on adiposity, insulin sensitivity, and hepatic steatosis in high-fat-fed mice. Am J Physiol Gastrointest Liver Physiol 292: G1671-G1682, 2007.

27. López-Parra M, Titos E, Horrillo R, et al: Regulatory effects of arachidonate 5-lipoxygenase on hepatic microsomal TG transfer protein activity and VLDL-triglyceride and apoB secretion in obese mice. J Lipid Res 49: 2513-2523, 2008.

28. Nozaki Y, Fujita K, Yoneda M, et al: Long-term combination therapy of ezetimibe and acarbose for non-alcoholic fatty liver disease. J Hepatol 51: 548-556, 2009.

29. Higuchi N, Kato M, Tanaka M, et al: Effects of insulin resistance and hepatic lipid accumulation on hepatic mRNA expression levels of apoB, MTP and L-FABP in non-alcoholic fatty liver disease. Exp Ther Med 2: 1077-1081, 2011.
30. Calvisi DF, Ladu S, Pinna F, et al: SKP2 and CKS1 promote degradation of cell cycle regulators and are associated with hepatocellular carcinoma prognosis. Gastroenterology 137: $1816-1826,2009$.

31. Gao D, Inuzuka H, Tseng A, Chin RY, Toker A and Wei W: Phosphorylation by Akt1 promotes cytoplasmic localization of Skp2 and impairs APCCdh1-mediated Skp2 destruction. Nat Cell Biol 11: 397-408, 2009.

32. Yang G, Ayala G, De Marzo A, et al: Elevated Skp2 protein expression in human prostate cancer: association with loss of the cyclin-dependent kinase inhibitor p27 and PTEN and with reduced recurrence-free survival. Clin Cancer Res 8: 3419-3426, 2002.

33. Li SH, Li CF, Sung MT, et al: Skp2 is an independent prognosticator of gallbladder carcinoma among p27(Kip1)-interacting cell cycle regulators: an immunohistochemical study of 62 cases by tissue microarray. Mod Pathol 20: 497-507, 2007.

34. Masuda TA, Inoue $\mathrm{H}$, Sonoda $\mathrm{H}$, et al: Clinical and biological significance of S-phase kinase-associated protein 2 (Skp2) gene expression in gastric carcinoma: modulation of malignant phenotype by Skp2 overexpression, possibly via p27 proteolysis. Cancer Res 62: 3819-3825, 2002.

35. Shigemasa K, Gu L, O'Brien TJ and Ohama K: Skp2 overexpression is a prognostic factor in patients with ovarian adenocarcinoma. Clin Cancer Res 9: 1756-1763, 2003.

36. Calvisi DF, Pinna F, Ladu S, et al: The degradation of cell cycle regulators by SKP2/CKS1 ubiquitin ligase is genetically controlled in rodent liver cancer and contributes to determine the susceptibility to the disease. Int J Cancer 126: 1275-1281, 2010.

37. Gstaiger M, Jordan R, Lim M, et al: Skp2 is oncogenic and overexpressed in human cancers. Proc Natl Acad Sci USA 98: 5043-5048, 2001.

38. Zhu L: Skp2 knockout reduces cell proliferation and mouse body size: and prevents cancer? Cell Res 20: 605-607, 2010.

39. Koga H, Harada M, Ohtsubo M, et al: Troglitazone induces p27Kip1-associated cell-cycle arrest through down-regulating Skp2 in human hepatoma cells. Hepatology 37: 1086-1096, 2003.

40. Signoretti S, Di Marcotullio L, Richardson A, et al: Oncogenic role of the ubiquitin ligase subunit Skp2 in human breast cancer. J Clin Invest 110: 633-641, 2002. 